\title{
ESTIMATES OF HAUSDORFF DIMENSION FOR NON-WANDERING SETS OF HIGHER DIMENSIONAL OPEN BILLIARDS
}

\author{
P. WRIGHT
}

\begin{abstract}
This article concerns a class of open billiards consisting of a finite number of strictly convex, non-eclipsing obstacles $K$. The non-wandering set $M_{0}$ of the billiard ball map is a topological Cantor set and its Hausdorff dimension has been previously estimated for billiards in $\mathbb{R}^{2}$, using well-known techniques. We extend these estimates to billiards in $\mathbb{R}^{n}$, and make various refinements to the estimates. These refinements also allow improvements to other results. We also show that in many cases, the non-wandering set is confined to a particular subset of $\mathbb{R}^{n}$ formed by the convex hull of points determined by period 2 orbits. This allows more accurate bounds on the constants used in estimating Hausdorff dimension.
\end{abstract}

\section{INTRODUCTION}

A billiard is a dynamical system in which a single pointlike particle moves at constant speed in some domain $Q \subset \mathbb{R}^{D}$ and reflects off the boundary $\partial Q$ according to the classical laws of optics [Ch. We describe a particle in the billiard by $x_{t}=$ $\left(q_{t}, v_{t}\right)$ where $q_{t} \in Q$ is the position of the particle and $v_{t} \in \mathbb{S}^{D-1}$ is its velocity at time $t$. Then for as long as the particle stays inside $Q$, it satisfies

$$
\left(q_{t+s}, v_{t+s}\right)=S_{s}\left(x_{t}\right)=\left(q_{t}+s v_{t}, v_{t}\right) .
$$

Collisions with the boundary are described by

$$
v^{+}=v^{-}-2\left\langle v^{-}, n\right\rangle n
$$

where $n$ is the normal vector (into $Q$ ) of $\partial Q$ at the point of collision, $v^{-}$is the velocity before reflection and $v^{+}$is the velocity after reflection.

Open billiards are a class of billiard in which the domain $Q$ is unbounded. We consider open billiards in which $Q=\mathbb{R}^{D} \backslash K$, where $K=K_{1} \cup \ldots \cup K_{u}$ is a union of pairwise disjoint, compact and strictly convex sets with $C^{2}$ boundary, for some integer $u \geq 3$. The $K_{i}$ are called obstacles. We assume that the no-eclipse condition $(\mathbf{H})$ holds. That is, for any nonequal $i, j, k$, the convex hull of $K_{i} \cup K_{j}$ does not intersect $K_{k}$. This condition ensures that the non-wandering set (defined later) does not include trajectories that are tangent to the boundary.

We denote by $n=n_{K}(q)$ the outward normal vector of $\partial K$ at $q$. Let $\hat{Q}=$ $\left\{(q, v) \in Q \times \mathbb{S}^{D-1} \mid q \in \operatorname{int} Q\right.$ or $\left.\langle n, v\rangle \geq 0\right\}$ be the phase space of $S_{t}$ with canonical projection $\pi: \hat{Q} \rightarrow Q$. Let $M=\left\{(q, v) \in \partial K \times \mathbb{S}^{D-1} \mid\langle n, v\rangle \geq 0\right\}$ be the boundary of $\hat{Q}$.

1991 Mathematics Subject Classification. Primary 37D20, Secondary 37D40.

Key words and phrases. Dynamical systems, billiards, dimension, Hausdorff. 
The non-wandering set of a dynamical system is the set of points whose trajectories never escape from the system i.e. the set of points $x$ such that the full trajectory $\left\{S_{t}(x): t \in \mathbb{R}\right\}$ is bounded. The non-wandering set of the flow is denoted $\Omega(S)$ or $\Omega$. Its restriction to the boundary is $M_{0}=\Omega \cap\left(\partial K \times S^{D-1}\right)$. Equivalently, $M_{0}=\left\{x \in M:\left|t_{j}(x)\right|<\infty\right.$ for all $\left.j \in \mathbb{Z}\right\}$, where $t_{j}(x) \in[-\infty, \infty]$ denotes the time of the $j$-th reflection of $x \in \hat{Q}$. Let $d_{j}(x)=t_{j}(x)-t_{j-1}(x)$. Let $\hat{Q}^{\prime}=t_{1}^{-1}(0, \infty)$, $M^{\prime}=M \cap \hat{Q}^{\prime}$ and define the billiard ball map as $B: M^{\prime} \rightarrow M, x \rightarrow S_{t_{1}(x)}(x)$. Then $B$ is invertible and $C^{2}$ (in general $B$ is at least as smooth as the boundaries of the obstacles), except where $v$ is tangent to $K$ at $B x$, and its restriction to $M_{0}$ is a bijection. $M_{0}$ is the non-wandering set of the billiard ball map; this non-wandering set is the main focus of this paper.

\section{Main Theorem}

The main result of this paper is in three parts.

Theorem 2.1. Let $K=K_{1} \cup \ldots \cup K_{u} \subset \mathbb{R}^{D}$ be disjoint, compact and strictly convex sets with smooth boundary, for some integer $u \geq 3$. Let $B$ be the billiard ball map in $Q=\mathbb{R}^{D} \backslash K$. Let $\lambda_{1}^{-1}=1+d_{\max } g_{\max }$ and $\mu_{1}^{-1}=1+d_{\min } g_{\min }$, where $d_{\min }$, $d_{\max }, g_{\min }$ and $g_{\max }$ are constants depending on the billiard, defined in Sections 3 and 11. Then the Hausdorff dimension of the non-wandering set $M_{0}$ of $B$ is given as follows:

(1) If $D=2$, then

$$
\frac{-2 \ln (u-1)}{\ln \lambda_{1}} \leq \operatorname{dim}_{H} M_{0} \leq \frac{-2 \ln (u-1)}{\ln \mu_{1}} .
$$

(2) If $D \geq 3$, and the obstacles $K_{i}$ are sufficiently far apart that $\lambda_{1}^{d_{\max }}<\mu_{1}^{2 d_{\min }}$, then equation (2.1) holds, although note that $g_{\min }$ is different in the higher dimensional case.

(3) We always have

$$
\alpha \frac{-2 \ln (u-1)}{\ln \lambda_{1}} \leq \operatorname{dim}_{H} M_{0} \leq \alpha^{-1} \frac{-2 \ln (u-1)}{\ln \mu_{1}},
$$

where $\alpha=\frac{2 d_{\min } \ln \mu_{1}}{d_{\max } \ln \lambda_{1}}$ is a particular Hölder constant, calculated in section 10.

Remark 2.2. Hassleblatt and Schmeling present a conjecture in [HS] that would imply that $\alpha=1$ for any billiard, making the above theorem much stronger. This will be discussed in section 9 .

Part 1 was essentially proved in $[\mathrm{Ke}$, except that the improvements to estimates in Section 11 can be applied. We deal with the higher dimensional case here.

\section{Properties of open Billiards}

The following lemma is well known (see for example [Sto2]).

Lemma 3.1. If $K$ satisfies the no-eclipse condition $(\boldsymbol{H})$, then for any finite sequence of indices $1 \leq i_{1}, \ldots, i_{n} \leq u(n \geq 3)$ such that $i_{j} \neq i_{j+1}$ for all $j$, let

$$
F: K_{i_{1}} \times \ldots \times K_{i_{n}} \rightarrow \mathbb{R},\left(q_{1}, \ldots, q_{n}\right) \mapsto \sum_{j=1}^{n}\left\|q_{j}-q_{j+1}\right\|
$$


where we denote $q_{n+1}=q_{1}$. Then $F$ achieves its minimum at some $\left(p_{1}, \ldots p_{n}\right)$ such that $p_{j} \in \partial K_{i_{j}}$ for all $j$. Specifically, the $p_{j}$ are the successive reflection points of a periodic billiard trajectory in $Q$ with $p_{j+1}=B p_{j}$ and $p_{1}=B p_{n}$.

\subsection{Billiard constants.}

Definition 3.2. At each point on a hypersurface $M$, the shape operator or second fundamental form (s.f.f.) $S_{p}: T_{p}(M) \rightarrow T_{p}(M)$ is defined by $S_{p}(v)=-\nabla_{v} n_{M}(p)$. The curvature of $\mathrm{M}$ at $p$ in the direction of a unit vector $\hat{u} \in T_{p}(M)$ is $k_{p}(\hat{u})=$ $S_{p}(\hat{u}) \cdot \hat{u}$.

Every billiard has several associated constants that can be useful in various estimates. The s.f.f $S_{q}(K)$ of $K$ at $q$ has $n-1$ eigenvalues, or principle curvatures. Let $\kappa_{\min }(q), \kappa_{\max }(q)$ denote the smallest and largest eigenvalues respectively at $q$. The billiard has minimum and maximum curvatures $\kappa^{-}=\min _{q \in \pi M_{0}} \kappa_{\min }(x)$ and $\kappa^{+}=$ $\max _{x \in M_{0}} \kappa_{\max }(x)$. We denote $d_{\min }=\min \left\{d_{i j}^{-}: 1 \leq i, j \leq u\right\}$ and $d_{\max }=\max \left\{d_{i j}^{-}\right.$: $1 \leq i, j \leq u\}$, where $d_{i j}^{-}$and $d_{i j}^{+}$are the respective minimum and maximum of the set $\left\{d(\pi x, \pi y): x \in K_{i} \cap M_{0}, y \in K_{j} \cap M_{0}\right\}$. For a point $x=(q, v) \in M$, we call $\phi(x)=\arccos \left\langle v, \nu_{K}(q)\right\rangle$ the collision angle, the acute angle which the $j$-th reflected ray makes with the outer normal to $K$. We denote $\phi_{j}(x)=\phi\left(B^{j} x\right)$. The collision angle can be bounded above by some constant $\phi^{+}=\max \left\{\phi(x): x \in M_{0}\right\}$. It can easily be shown that $\phi^{+} \leq \arccos \left(b^{-} / d_{\max }\right)$, where $b^{-}=\min _{i, j, k} d\left(K_{j}, \operatorname{Cvx}\left(K_{i}, K_{k}\right)\right)$.

\section{Convex fronts}

Let $X$ be a smooth, stricly convex $D-1$ dimensional surface in int $Q$ with outer normal field $v(q)$, let $\hat{X}=\{(q, v(q)): q \in X\}, \hat{X}_{0}=\hat{X} \cap \Omega$ and $X_{0}=\pi \hat{X}_{0}$ where $\pi$ is the canonical projection. Let $x, y \in X$. Let $Y: q(s), s \in[0,1]$ be a $C^{3}$ curve on $X$ with outer normal field parametrised by $v(s)=v(q(s))$. Let $Y_{0}=Y \cap X_{0}$, $\hat{X}_{t}=S_{t}(\hat{X}), X_{t}=\pi \hat{X}_{t}, \hat{Y}_{t}=S_{t}(\hat{Y}), Y_{t}=\pi \hat{Y}_{t}$ and $t_{j}(s)=t_{j}(q(s), n(s))$. Where defined, let $q_{j}(s)=\pi B^{j}(q(s), v(s))$ be the $j$-th reflection point of $(q(s), v(s))$, then let $d_{j}(s)=t_{j}(s)-t_{j-1}(s)$, and $\phi_{j}(s)=\phi_{j}(q(s), v(s))$.

For a point $q \in \partial K$, let $\mathcal{J}$ denote the tangent space $T_{q}(X)$ of the convex front, and let $\mathcal{T}$ denote the tangent space of $\partial K$ at $q$. The s.f.f of $X$ at $q$ is given by $\mathcal{B}: \mathcal{J} \rightarrow \mathcal{J}, \mathcal{B} d v=S_{q}(d v)$.

4.1. Evolution of Fronts. With no collisions, the curvature of a convex front $X$ front is given by the formula BCST]

$$
\mathcal{B}\left(q_{t}(s)\right)=\left(\mathcal{B}(q)^{-1}+t I\right)^{-1} .
$$

At a collision point, let $B^{-}$be the second fundamental form just before the collision and let $\mathcal{B}^{+}$be the s.f.f. just after the collision. Then

$$
\mathcal{B}^{+}=\mathcal{B}^{-}+2 \Theta=\mathcal{B}^{-}+2\langle n, v\rangle V^{*} K V,
$$

where $V: \mathcal{J} \rightarrow \mathcal{T}$ is the projection $V d v=d v-\frac{\langle d v, n\rangle}{\langle n, v\rangle} v \in \mathcal{T}, K: \mathcal{T} \rightarrow \mathcal{T}$ is the s.f.f. of $K$ at $q, V^{*}: \mathcal{T} \rightarrow \mathcal{J}$ is the projection $V^{*} d q=d q-\frac{\langle d q, v\rangle}{\langle n, v\rangle} n \in \mathcal{J}$, and $\langle n, v\rangle=\cos \phi$ where $\phi \in\left[0, \frac{\pi}{2}\right]$ is the collision angle. 


\subsection{Estimating $\Theta$.}

Lemma 4.1. If the dimension $n$ is greater than 2, let $\kappa_{\min }$, $\kappa_{\max }$ be the smallest and largest eigenvalues of the s.f.f. $K$ at $q$, so that $\kappa_{\min }|d q| \leq\|K d q\| \leq \kappa_{\max }|d q|$. Then

$$
\kappa_{\min } \cos \phi \leq\|\Theta\| \leq \frac{\kappa_{\max }}{\cos \phi}
$$

Proof. If $n=v$ then $\langle n, v\rangle=\cos \phi=1$ so $\Theta=\langle n, v\rangle V^{*} K V=K$ and the inequality holds. Henceforth we assume $n \neq v$. Let $\mathcal{S}=\mathcal{J} \cap \mathcal{T}$. Any vector $d v \in \mathcal{J}$ can be written in the form $d v=|d v|(\hat{a} \cos \theta+\hat{s} \sin \theta)$, where $\hat{s} \in \mathcal{S}$ and $\hat{a} \in \mathcal{J}$ are unit vectors, $\hat{a}$ is perpendicular to $\mathcal{S}$, and $\langle\hat{a}, n\rangle \geq 0$. Then $\hat{a}$ is in the plane containing by $n$ and $v$ so the angle between $\hat{a}$ and $n$ is $\frac{\pi}{2}-\phi$. Using $d v \perp v$ we get

$$
\begin{aligned}
\|V d v\| & =\left\|d v-\frac{\langle|d v| \hat{s} \sin \theta, n\rangle}{\langle n, v\rangle} v-\frac{\langle|d v| \hat{a} \cos \theta, n\rangle}{\langle n, v\rangle} v\right\| \\
& =\|d v-(|d v| \tan \phi \cos \theta) v\| \\
& =\sqrt{1+\tan ^{2} \phi \cos ^{2} \theta}|d v|
\end{aligned}
$$

Similarly, write $d q \in \mathcal{T}$ as $d q=|d q|\left(\left(\hat{b} \cos \theta^{\prime}+\hat{s}^{\prime} \sin \theta^{\prime}\right)\right.$ for some unit vectors $\hat{s^{\prime}} \in \mathcal{S}$ and $\hat{b} \in \mathcal{T}$ with $\hat{b} \perp \mathcal{S}$ and $\langle\hat{b}, \hat{v}\rangle \geq 0$. Then $\left\|V^{*} d q\right\|=\sqrt{1+\tan ^{2} \phi \cos ^{2} \theta^{\prime}}|d q|$. Combining these operator norms and using $0 \leq \cos ^{2} \theta, \cos ^{2} \theta^{\prime} \leq 1$, we get

$$
\begin{aligned}
\kappa_{\min } \cos \phi & \leq \cos \phi \sqrt{1+\tan ^{2} \phi \cos ^{2} \theta} \kappa_{\min } \sqrt{1+\tan ^{2} \phi \cos ^{2} \theta^{\prime}} \\
& \leq\|\Theta\| \leq \cos \phi \sqrt{1+\tan ^{2} \phi \cos ^{2} \theta} \kappa_{\max } \sqrt{1+\tan ^{2} \phi \cos ^{2} \theta^{\prime}} \\
& \leq \cos \phi \kappa_{\max }\left(1+\tan ^{2} \phi\right) \leq \frac{\kappa_{\max }}{\cos \phi}
\end{aligned}
$$

as required.

Note that in the two dimensional case, $\theta=\theta^{\prime}=0$ since $\mathcal{S}=\mathcal{T} \cap \mathcal{J}=\{q\}$, and $\kappa_{\min }=\kappa_{\max }=\kappa$ at every point. So the inequality becomes $\|\Theta\|=\frac{\kappa}{\cos \phi}$.

4.3. Estimating $k_{j}$. This section follows the definitions in Sto1]. Let $u_{j}(s)=$ $\lim _{\tau \downarrow t_{j}(s)} \frac{d}{d s} S_{\tau} q(s)$ and let $\hat{u}_{j}(s)=\frac{u_{j}(s)}{\left\|u_{j}(s)\right\|}$ be the unit tangent vector of $Y_{t}$ at $q(s)$. Let $\mathcal{B}_{j}$ be the s.f.f. of $S_{t_{j}(s)} X$ at $q_{j}(s)$. Define $\ell_{j}(s)>0$ by

$$
\left[1+d_{j}(s) \ell_{j}(s)\right]^{2}=\left\|\hat{u}_{j}(s)+d_{j}(s) \mathcal{B}_{j} \hat{u}_{j}(s)\right\|^{2},
$$

Then set $\delta_{j}(s)=\frac{1}{1+d_{j}(s) \ell_{j}(s)}$.

Proposition 4.2. Fix a point $x_{0}=\left(q_{0}, v_{0}\right) \in \hat{X}$, a positive integer $m$ and some $\tau$ with $t_{m}\left(x_{0}\right)<\tau<t_{m+1}\left(x_{0}\right)$. Let $Y:[0, a] \rightarrow X$ be a $C^{3}$ curve with $q(0)=q_{0}$ with a small enough that for every $s \in[0, a]$ we have $t_{m}(x(s))<\tau<t_{m+1}(x(s))$, where $x(s)=\left(q(s), \nu_{X}(q(s))\right)$, and that for all $j=1, \ldots, m$ the points $q_{j}(s) \in \partial K_{i_{j}}$ for all $s \in[0, a]$. Then $p(s)=\pi S_{t}(x(s))$ is a $C^{3}$ curve on $X_{t}$. For all $s \in[0,1]$ we have

$$
\left\|q^{\prime}(s)\right\|=\frac{\left\|p^{\prime}(s)\right\|}{\left.1+\left(\tau-t_{m}(s)\right) k_{m}(s)\right)} \delta_{0}(s) \delta_{1}(s) \ldots \delta_{m}(s) .
$$


Proof. See [Sto1, Sto3]. The same result can be derived from [BCST], and is also proved for completeness (in two dimensions only) in [Ke].

Now the curvature of the convex front after $j$ reflections in the direction $\hat{u}_{j}$ is $k_{j}=\left\langle\mathcal{B}_{j} \hat{u}_{j}, \hat{u}_{j}\right\rangle$, so

$$
1 / \delta_{j}(s)^{2}=1+2 d_{j}(s) k_{j}(s)+d_{j}(s)^{2}\left\|\mathcal{B}_{j} \hat{u}_{j}(s)\right\|^{2} .
$$

Let $q \in X$ and let $x=\left(q, \nu_{X}(q)\right)$. Let $\mu_{j}(s)$ and $\lambda_{j}(s)$ be the minimum and maximum eigenvalues of $\mathcal{B}_{j}(q(s))$ respectively.

Recall that $\mathcal{B}_{j+1}=\mathcal{B}_{j+1}^{-}+2 \Theta=\left(\mathcal{B}_{j}^{-1}+d_{j} I\right)^{-1}+2 \Theta . \quad \mathcal{B}_{j}$ is always positive definite, so $\mu_{j}$ and $\lambda_{j}$ are always positive. Note that if $\lambda$ is an eigenvalue of $\mathcal{B}(q(s))$, then $\frac{\lambda}{1+t \lambda}$ is an eigenvalue of $\mathcal{B}\left(q_{t}(s)\right)$. So we have $\lambda_{j+1}=\frac{\lambda_{j}}{1+d_{j} \lambda_{j}}+\frac{2 \kappa_{\max }\left(x_{j}\right)}{\cos \phi_{j}(x)}$ and $\mu_{j+1}=\frac{\mu_{j}}{1+d_{j} \mu_{j}}+2 \kappa_{\max }\left(x_{j}\right) \cos \phi_{j}(x)$. For all $j \geq 0, \mu_{j}(s) \leq k_{j}(s) \leq \lambda_{j}(s)$, so we get $k_{j+1}(s) \in$

$$
\left[\frac{k_{j}(s)}{1+d_{j}(s) k_{j}(s)}+2 \kappa_{\min }\left(x_{j}(s)\right) \cos \phi_{j}(s), \frac{k_{j}(s)}{1+d_{j}(s) k_{j}(s)}+\frac{2 \kappa_{\max }\left(x_{j}(s)\right)}{\cos \phi_{j}(s)}\right] .
$$

\section{CODIng $M_{0}$ AND $X_{0}$}

For each $x \in M_{0}$ we have a bi-infinite sequence of indices $\alpha=\left\{\alpha_{i}\right\}_{i=-\infty}^{\infty}, \alpha_{i} \in$ $\{1, \ldots, u\}$ such that $\pi B^{i} x \in \partial K_{\alpha_{i}}$. Since each $K_{i}$ is convex, $\alpha_{i} \neq \alpha_{i+1}$ for all $i$, so define the symbol spaces $\Sigma$ and $\Sigma^{+}$as

$$
\begin{gathered}
\Sigma=\left\{\left(\alpha_{i}\right)_{i=-\infty}^{\infty}: \alpha_{i} \in\{1, \ldots, u\}, \alpha_{i} \neq \alpha_{i+1} \text { for all } i \in \mathbb{Z}\right\}, \\
\Sigma^{+}=\left\{\left(\alpha_{i}\right)_{i=1}^{\infty}: \alpha_{i} \in\{1, \ldots, u\}, \alpha_{i} \neq \alpha_{i+1} \text { for all } i \leq 0\right\} .
\end{gathered}
$$

Let $f: M_{0} \rightarrow \Sigma, x \mapsto \alpha$ denote the representation map. The two-sided subshift $\sigma: \Sigma \rightarrow \Sigma, \alpha_{i} \mapsto \alpha_{i+1}$ is continuous under the following metric $d_{\theta}$ for any $\theta \in(0,1)$.

$$
d_{\theta}(\alpha, \beta)= \begin{cases}0: & \text { if } \alpha_{i}=\beta_{i} \text { for all } i \in \mathbb{Z} \\ \theta^{n}: & \text { if } n=\max \left\{j \geq 0: \alpha_{i}=\beta_{i} \text { for all }|i|<j\right\},\end{cases}
$$

We define a similar metric on $\Sigma^{+}$.

$$
d_{\theta}(\alpha, \beta)= \begin{cases}0: & \text { if } \alpha_{i}=\beta_{i} \text { for all } i \geq 0 \\ \theta^{n}: & \text { if } n=\max \left\{j \geq 0: \alpha_{i}=\beta_{i} \text { for all } 0 \leq i \leq j\right\},\end{cases}
$$

Lemma 5.1. If $u \geq 2$ and $\theta \in(0,1)$, then $f$ is a homeomorphism of $M_{0}$ (with the topology induced by $M)$ onto $\left(\Sigma, d_{\theta}\right)$, and the shift $\sigma$ is topologically conjugate to $B$, that is $B=f^{-1} \circ \sigma \circ f$.

Assuming $u \geq 3, M_{0}$ is a compact topological Cantor set. $B$ is topologically transitive on $M_{0}$ and its periodic points are dense in $M_{0}$. B is hyperbolic on $M_{0}$, and $M_{0}$ is a basic set for $B$.

Given the surface $X$, the intersection $X_{0}=X \cap \Omega$ can also be coded by sequences. Define the representation map $\Upsilon: X_{0} \rightarrow \Sigma^{+}$in the same way as $f: M_{0} \rightarrow \Sigma$. Define an equivalence relation $\sim_{m}(m \geq 0)$ by $\alpha \sim_{m} \beta \Leftrightarrow \alpha_{i}=\beta_{i}$ for all $1 \leq i \leq m$, and $\alpha \sim_{0} \beta$ for any $\alpha, \beta \in \Sigma^{+}$. We call the equivalence classes $[\alpha]_{m}$ cylinders. Define another relation (not an equivalence relation) $\approx_{m}$ by $\alpha \approx_{m} \beta$ if $\alpha \sim_{m} \beta$ and $\alpha_{m+1} \neq \beta_{m+1}$. 
The following lemma on Hausdorff dimension and packing dimension is the result of direct calculations (see for example $[\mathrm{Ed}, \mathrm{Ke}]$ ).

Lemma 5.2. For any $\alpha \in \Sigma^{+}$and $N \in \mathbb{N}$,

$$
\overline{\operatorname{dim}_{p}}\left([\alpha]_{N}, d_{\theta}\right)=\operatorname{dim}_{H}\left([\alpha]_{N}, d_{\theta}\right)=\frac{-\ln (u-1)}{\ln \theta} .
$$

We find upper and lower bounds $g_{\min }$ and $g_{\max }$ such that for some $N \in \mathbb{N}$, $k_{j}(s) \in\left[g_{\min }, g_{\max }\right]$ for all $j \geq N$.

\section{Estimating $\delta_{j}(s)$}

Section 4.1 of $\mathrm{Ke}$ contains a significant improvement to the dimension estimate using the continued fraction for $k_{j}(s)$. We can do the same using the bounds in (4.1).

The map $f_{\gamma, \theta}:(0, \infty) \rightarrow \mathbb{R}, x \mapsto \frac{x}{1+\theta x}+2 \gamma$ has one positive fixed point $g(\gamma, \theta)=$ $\gamma+\sqrt{\gamma^{2}+2 \gamma / \theta}$. This function is non-decreasing in $\gamma$ and strictly decreasing in $\theta$.

The natural domain for $g$ is $\left[\kappa_{\min } \cos \phi^{+}, \frac{\kappa_{\max }}{\cos \phi^{+}}\right] \times\left[d_{\min }, d_{\max }\right]$ for the arguments of $g$. On this domain, the minimum and maximum values of $g$ are $g\left(\kappa_{\min }, d_{\max }\right)$ and $g\left(\frac{\kappa_{\max }}{\cos \phi^{+}}, d_{\min }\right)$ respectively. While this domain is an obvious choice, it is not the strictest or most useful domain. We will use a smaller domain $\mathbb{D}$ defined in Section 11

We write $g_{\min }=\max _{(\gamma, \theta) \in \mathbb{D}} g(\gamma, \theta)$ and $g_{\max }=\max _{(\gamma, \theta) \in \mathbb{D}} g(\gamma, \theta)$. The values that maximise and minimise $g$ are denoted $\left(\gamma_{\max }, \theta_{\min }\right)$ and $\left(\gamma_{\min }, \theta_{\max }\right)$ respectively.

Parametrise the surface $X$ by $q(t)=q\left(t_{1}, \ldots, t_{D-1}\right)$ where each $t_{i} \in[0,1]$ and $D$ is the dimension of the billiard. Let $U T(X)=\{(q, \hat{u}): q \in X,\|\hat{u}\|=$ $1, \hat{u}$ tangent to $X$ at $q\}$ denote the unit tangent bundle of $X$, and parametrise $U T(X)$ by $x(s)=x(t, \hat{u})$, where $s \in[0,1]^{D-1} \times \mathbb{S}^{D-2}$. Consider any $s=(t, \hat{u}) \in S$ such that $q(t) \in X_{0}$ and any sequences $\left(\gamma_{j}, \theta_{j}\right)_{1}^{\infty} \subset \mathbb{D}$. Let $k_{0}(s)=\mathcal{B}_{0}(t)(\hat{u}) \cdot \hat{u}$ be the curvature of $X$ at $q(t)$ in the direction $\hat{u}$, and inductively define $k_{j+1}(s)=$ $f_{\gamma_{j}, \theta_{j}}\left(k_{j}(s)\right)$ for $0 \leq j \leq n-1$.

Lemma 6.1. Let $a<g_{\min }$ and $b>g_{\max }$. Then there exists $n(X)>0$ such that for all $s$ and $j \geq n(X)$ we have $k_{j}(s) \in[a, b]$.

Proof. If $k_{N}(s) \leq g_{\max }$ for some $s$ and some $N \geq 0$ then inductively

$$
k_{j+1}(s)=f_{\gamma_{j}, \theta_{j}}\left(k_{j}(s)\right) \leq f_{\gamma_{\max }, \theta_{\min }}\left(k_{j}(s)\right) \leq f_{\gamma_{\max }, \theta_{\min }}\left(g_{\max }\right)=g_{\max }
$$

for all $j \geq N$. Similarly if $k_{N}(s) \geq g_{\min }$ for some $N$ then $k_{j}(s) \geq g_{\min }$ for all $j \geq N$. For each $s$, define $k_{j}^{-}$and $k_{j}^{+}$by $k_{0}^{-}=k_{0}, k_{j+1}^{-}=f_{\gamma_{\min }, \theta_{\max }}\left(k_{j}^{-}\right)$and $k_{0}^{+}=k_{0}, k_{j+1}^{+}=f_{\gamma_{\max }, \theta_{\min }}\left(k_{j}^{+}\right)$. Then for all $j \geq 0$ and $s \in S$ we have $k_{j}^{-}(s) \leq$ $k_{j}(s) \leq k_{j}^{+}(s), \lim _{j \rightarrow \infty} k_{j}^{-}(s)=g_{\min }$ and $\lim _{j \rightarrow \infty} k_{j}^{+}(s)=g_{\max }$. There must be some integer $j_{0}(s) \geq 0$ such that $k_{j}(s) \in[a, b]$ for all $j \geq j_{0}(s)$.

Since $T X$ is compact, $k_{0}(s)$ has an infimum $k_{0, \min }=k_{0}\left(s_{\min }\right)$ and a supremum $k_{0, \max }=k_{0}\left(s_{\max }\right)$. Let $n(X)=\max \left\{j_{0}\left(s_{\min }\right), j_{0}\left(s_{\max }\right)\right\}$. Then for $j \geq n(X)$,

$$
a \leq k_{j}\left(s_{\min }\right) \leq k_{j}(s) \leq k_{j}\left(s_{\max }\right) \leq b,
$$

so $j_{0}(s) \leq n(X)$ for all $s \in S$. Thus we have $k_{j}(s) \in[a, b]$ for all $j \geq n(X)$ as required. 
For any $\tau \geq 0, n(X) \geq n\left(S_{\tau} X\right)$. So by taking a finite number of convex fronts $X_{i}$ whose image under $S_{\tau}$ covers $\Omega$, we can get a global constant $n_{0}=n(a, b)=$ $\max \left\{n\left(X_{i}\right): M \subset \bigcup_{i} X_{i}\right\}$ that depends only on $a, b$ and the billiard itself.

Now $k_{j}(s) \in(a, b)$ for all $s \in q^{-1}(X)$ and $j>n_{0}$. So for these values,

$$
\delta_{j}(s) \in\left(\frac{1}{1+d_{\max } b}, \frac{1}{1+d_{\min } a}\right) .
$$

Define $\lambda=\frac{1}{1+d_{\max } b}$ and $\mu=\frac{1}{1+d_{\min } a}$ for now. For $0 \leq j<n_{0}$, we can still find bounds for $\delta_{j}(s) . k_{j}(s)$ is always bounded below by 0 , and we can assume $k_{0}(s)$ is bounded above by some $k_{0}^{+}\left[\underline{\mathrm{S}}\right.$. So $\delta_{j}(s) \in\left[\delta^{-}, 1\right]$ where $\delta^{-}=\frac{1}{1+d_{\max } k_{0}^{+}}$. Furthermore, we have $2 \kappa^{-} \cos \phi^{+} \leq k_{j}(s) \leq \frac{1}{d_{\min }}+\frac{2 \kappa^{+}}{\cos \phi^{+}}$for $1 \leq j<n_{0}$. Thus, $\delta_{j}(s) \in\left[\lambda_{0}, \mu_{0}\right]$ where $\lambda_{0}^{-1}=1+d_{\max }\left(\frac{1}{d_{\min }}+\frac{2 \kappa^{+}}{\cos \phi^{+}}\right)$and $\mu_{0}^{-1}=1+2 d_{\min } \kappa^{-} \cos \phi^{+}$.

\section{HAUSDORFF DIMENSION OF $X_{0}$}

Proposition 7.1. Let $[a, b] \supset\left[g_{\min }, g_{\max }\right], \lambda=\frac{1}{1+d_{\max } b}, \mu=\frac{1}{1+d_{\min } a}$, and $n_{0}=$ $n(a, b)$ as defined above. There exist constants $c, C$ depending only on the billiard, such that for any integer $n \geq n_{0}$ and $x_{1}, x_{2} \in \hat{X}_{0}$ such that $x_{1} \approx_{n} x_{2}$, we have

$$
c \lambda^{n-n_{0}} \leq\left\|\pi x_{1}-\pi x_{2}\right\| \leq C \mu^{n-n_{0}} .
$$

Proof. Let $n \geq n_{0}$ and let $x_{1}, x_{2} \in \hat{X}_{0}$ with $x_{1} \approx_{n} x_{2}$. Without loss of generality assume $t_{n}\left(x_{1}\right)<t_{n}\left(x_{2}\right)$ and let $\tau=t_{n}\left(x_{2}\right)$. Let $y_{1}=S_{\tau} x_{1}, y_{2}=S_{\tau} x_{2}$. Now let $p(s)$ parametrize (by arc length) the shortest curve $\Gamma \subset S_{\tau} X$ between $y_{1}$ and $y_{2}$. Let $q(s)=S_{-\tau}(p(s))$ paramatrize the curve $Y=S_{-\tau} \Gamma$. This curve will not be the shortest curve between its endpoints $x_{1}$ and $x_{2}$, in fact for large $n$ it can be much longer. We have

$$
\begin{aligned}
\left\|\pi x_{1}-\pi x_{2}\right\| & =\left\|\int_{Y} q^{\prime}(s) d s\right\| \leq \int_{Y}\left\|q^{\prime}(s)\right\| d s \\
& =\int_{\Gamma} \frac{\left\|p^{\prime}(s)\right\|}{1+\left(\tau-t_{n}(s)\right) k_{n}(s)}\left(\prod_{j=0}^{n-1} \delta_{j}(s)\right) d s \\
& \leq \mu^{n-n_{0}} \mu_{0}^{n_{0}} \int_{\Gamma} d s \leq C \mu^{n-n_{0}} .
\end{aligned}
$$

Here we used Proposition 4.2, $\left(\tau-t_{n}(s)\right) k_{n}(s) \geq 0, \delta_{j}(s)<\mu_{0}$ for $0 \leq j \leq n_{0}$, $\delta_{j}(s)<\mu$ for $j>n_{0}$. Since the curve $\Gamma$ is the shortest curve between two points on a surface with bounded curvature [S], and confined to a bounded set (e.g. a ball containing $K$ ), its arc length $\int_{\Gamma} d s$ can be bounded above by a constant.

Now we find an estimate for $\left\|x_{1}-x_{2}\right\|$ from below, using different curves. Let $q(s)$ parametrise the shortest curve $Y$ in $X$ between $x_{1}$ and $x_{2}$. Now let $\left[s_{1}, s_{2}\right] \subseteq[0,1]$ such that $s=s_{1}, s_{2}$ are the only values for which $(q(s), n(s))$ has an $(n+1)$-st reflection. Let $y_{1}=q_{n+1}\left(s_{1}\right), y_{2}=q_{n+1}\left(s_{2}\right)$. Without loss of generality assume $t_{n+1}\left(s_{1}\right)<t_{n+1}\left(s_{2}\right)$ and let $\tau=t_{n+1}\left(s_{1}\right), z=S_{\tau}\left(q\left(s_{2}\right)\right)$. Then $p(s)=S_{\tau} q(s)$ parametrizes the curve $S_{\tau} \hat{Y}$. 
We have constants $C_{1}$ and $C_{2}$ such that

$$
\begin{aligned}
\left\|\pi x_{1}-\pi x_{2}\right\| & \geq C_{1} \int_{X}\left\|q^{\prime}(s)\right\| d s \geq C_{1} \int_{s_{1}}^{s_{2}}\left\|q^{\prime}(s)\right\| d s \\
& =C_{1} \int_{s_{1}}^{s_{2}} \frac{\left\|p^{\prime}(s)\right\|}{1+\left(\tau-t_{n}(s)\right) k_{n}(s)}\left(\prod_{j=0}^{n-1} \delta_{j}(s)\right) \\
& \geq C_{1} C_{2} \lambda_{0}^{n_{0}} \lambda^{n-n_{0}} \int_{s_{1}}^{s_{2}}\left\|p^{\prime}(s)\right\| d s
\end{aligned}
$$

Clearly $z$ is in the convex hull of the two obstacles containing $q_{n}\left(s_{2}\right)$ and $y_{2}$ respectively, and $y_{1}$ is in a third obstacle. Thus we have $\int_{s_{1}}^{s_{2}}\left\|p^{\prime}(s)\right\| \geq\left\|y_{1}-z\right\| \geq b^{-}$, where $b^{-}$is the minimum distance between $K_{k}$ and $\operatorname{Cvx}\left(K_{i} \cup K_{j}\right)$ for any nonequal $i, j, k$. Letting $c=C_{1} C_{2} \lambda_{0}^{n_{0}} b^{-}$, we have $c \lambda^{n-n_{0}} \leq\|\pi x-\pi y\| \leq C \mu^{n-n_{0}}$ as required.

Proposition 7.2. Let $0<n_{0} \leq n$. Suppose there are constants $c, C>0$ such that $c \lambda^{n-n_{0}} \leq\|\pi x-\pi y\| \leq C \mu^{n-n_{0}}$ whenever $x, y \in \hat{Y}_{0}$ with $x \approx_{n} y$. Then $\Upsilon: \hat{Y}_{0} \rightarrow \Sigma^{+}$ is injective and a Lipschitz homeomorphism from $\hat{Y}_{0}$ to the metric space $\left(\Upsilon\left(\hat{Y}_{0}\right), d_{\lambda}\right)$, and $\Upsilon^{-1}$ is a Lipschitz homeomorphism from $\left(\Upsilon\left(\hat{Y}_{0}\right), d_{\mu}\right)$ onto $\hat{Y}_{0}$.

Proof. For any $x \in X_{0}$ with sufficiently large $n \geq n_{0}$, there is some $z \in X_{0}$ such that $z \approx_{n} x$, so if $\Upsilon(x)=\Upsilon(y)$ then $\|x-y\| \leq\|x-z\|+\|y-z\| \leq 2 C \mu^{n} \rightarrow 0$ as $n \rightarrow \infty$. So $\Upsilon^{-1}$ is well defined and $\Upsilon$ is injective.

Let $x \approx_{n} y \in X_{0}$. Then $d_{\lambda}(\Upsilon x, \Upsilon y)=\lambda^{n} \leq \frac{1}{c}\|x-y\|$, so $\Upsilon$ is Lipschitz.

Similarly, for distinct $\alpha, \beta \in \Upsilon\left(X_{0}\right), x \in \Upsilon^{-1}(\alpha), y \in \Upsilon^{-1}(\beta)$, and $n$ such that $x \approx_{n} y \in X_{0}$, we have $\left\|\Upsilon^{-1}(\alpha)-\Upsilon^{-1}(\beta)\right\| \leq C \mu^{n}=C d_{\mu}(\alpha, \beta)$. Finally, since the identity $I:\left(\Upsilon\left(X_{0}\right), d_{\lambda}\right) \rightarrow\left(\Upsilon\left(X_{0}\right), d_{\mu}\right)$ is continuous, the maps $\Upsilon: X_{0} \rightarrow$ $\left(\Upsilon\left(X_{0}\right), d_{\mu}\right)$ and $\Upsilon^{-1}:\left(\Upsilon\left(X_{0}\right), d_{\lambda}\right) \rightarrow X_{0}$ are also continuous.

The following theorem is well known (see $\mathrm{Fa}$ )

Theorem 7.3. Let $f: A \rightarrow B$ be a Lipschitz map and let $F \subset A$. Then $\operatorname{dim}_{H} f(F) \leq \operatorname{dim}_{H} F$.

For some $\alpha \in \Sigma^{+}$and sufficiently large $n \geq n_{0}$ the cylinder $[\alpha]_{n} \subset \Upsilon\left(\hat{Y}_{0}\right)$. It follows that $\operatorname{dim}_{H}\left(\Upsilon\left(\hat{Y}_{0}\right), d_{\lambda}\right) \leq \operatorname{dim}_{H} \hat{Y}_{0} \leq \operatorname{dim}_{H}\left(\Upsilon\left(\hat{Y}_{0}\right), d_{\mu}\right)$.

\section{HausdorfF Dimension of $M_{0}$}

We now relate $\operatorname{dim}_{H} X_{0}$ to $\operatorname{dim}_{H} M_{0}$. Let $x \in M_{0}$ and let $\hat{X}=S_{\tau}\left(W_{\theta}^{(u)}(x)\right)$ be the image of the local unstable manifold $W_{\theta}^{(u)}(x)$ under $S_{t}$. Let $X_{0}=X \cap M_{0}$. Define $d^{(s)}=\operatorname{dim}_{H}\left(W_{\theta}^{(s)}(x) \cap M_{0}\right)$ and $d^{(u)}=\operatorname{dim}_{H}\left(W_{\theta}^{(u)}(x) \cap M_{0}\right)$. Then using Lemma 5.2 we get

$$
d^{(u)}=\operatorname{dim}_{H} X_{0} \in\left[\frac{-\ln (u-1)}{\ln \lambda}, \frac{-\ln (u-1)}{\ln \mu}\right] .
$$

We can use the same estimate for $d^{(s)}$, since $W_{\theta}^{(u)}=\operatorname{Refl} W^{(s)}(\operatorname{Refl}(x))$, where Refl: $\hat{Q} \rightarrow \hat{Q}$ is a bi-Lipschitz involution given by 


$$
\operatorname{Refl}(q, v)=\left\{\begin{array}{lr}
(q,-v) & \text { for } q \in \operatorname{int} Q \\
\left.\left(q, 2\left\langle n_{K}(q), v\right\rangle n_{K}(q)-v\right\rangle\right), & \text { for } q \in \partial K
\end{array}\right.
$$

If $E, F$ are Borel sets, the following inequalities are well known (see [Fa]).

$$
\operatorname{dim}_{H} E+\operatorname{dim}_{H} F \leq \operatorname{dim}_{H}(E \times F) \leq \operatorname{dim}_{H} E+\overline{\operatorname{dim}_{p}} F .
$$

Lemma 5.2 gives $\overline{\operatorname{dim}_{p}}\left(\Sigma^{+}, d_{\theta}\right)=\operatorname{dim}_{H}\left(\Sigma^{+}, d_{\theta}\right)$. Let $V$ be a neighbourhood of $M_{0}$ and let $U \subset V$ be a neighbourhood of $x$. Let $\varepsilon$ be small enough that $W_{\varepsilon}^{(u)}(x), W_{\varepsilon}^{(s)}(x) \subset U$, and let $h: W_{\varepsilon}^{(u)}(x) \times W_{\varepsilon}^{(s)}(x) \rightarrow R$ be the usual local product map, where $R$ is an open neighbourhood of $x$. This holonomy is at least Hölder continuous. Let $\alpha$ be the Hölder constant of $h$, then using basic properties of Hausdorff dimension $\mathrm{Fa}$ ] we have

$$
\alpha\left(d^{(s)}+d^{(u)}\right) \leq \operatorname{dim}_{H}\left(R \cap M_{0}\right) \leq \alpha^{-1}\left(d^{(s)}+d^{(u)}\right) .
$$

If $\alpha=1$ we have

$$
\operatorname{dim}_{H}\left(R \cap M_{0}\right)=d^{(s)}+d^{(u)} .
$$

Theorem 8.1. Let $\lambda_{1}=\frac{1}{1+d_{\max } g_{\max }}, \mu_{1}=\frac{1}{1+d_{\min } g_{\min }}$. Assume that $\alpha=1$. Then

$$
\frac{-2 \ln (u-1)}{\ln \lambda_{1}} \leq \operatorname{dim}_{H} M_{0} \leq \frac{-2 \ln (u-1)}{\ln \mu_{1}} .
$$

Proof. For any $a<g_{\min }, b>g_{\max }$, letting $\lambda(b)=\frac{1}{1+d_{\max } b}, \mu(a)=\frac{1}{1+d_{\min } a}$ we have

$$
\operatorname{dim}_{H} M_{0}=\operatorname{dim}_{H}\left(R \cap M_{0}\right)=d^{(s)}+d^{(u)} \in\left[\frac{-2 \ln (u-1)}{\ln \lambda(b)}, \frac{-2 \ln (u-1)}{\ln \mu(a)}\right] .
$$

Taking limits $a \rightarrow g_{\min }$ and $b \rightarrow g_{\max }$, we get the result.

\section{Dimension product StRuCture}

In this section we discuss what is currently known about the holonomy $h$. The holonomy is always Lipshitz if the diffeomorphism $B$ is conformal on both the stable and unstable manifolds (see $[\mathrm{B}$ and $\S 7$ of $[\mathrm{P}]$ ). This is the case for the billiard ball map $B$ in $\mathbb{R}^{2}$ but not in higher dimensions. To see this, suppose one of the obstacles is the unit sphere centered on the origin, and consider an unstable manifold containing the points $(0,0,10),\left(\frac{1}{2}, 0,10\right),\left(0, \frac{1}{2}, 10\right)$, each with a ray in a direction sufficiently close to $(0,0,-1)$ that the rays collide with the sphere. These points form a right angle, but their image under $B$ does not, so $B$ does not always preserve angles on unstable manifolds and is not conformal.

However Stoyanov in Sto1 showed that a class of billiards satisfy a pinching condition, which would imply the stable and unstable manifolds are $C^{1}$. In the notation of this paper, a billiard satisfies the pinching condition if $\lambda_{0}^{d_{\max }}<\mu_{0}^{2 d_{\min }}$, where $\lambda_{0}^{-1}=1+d_{\max }\left(\frac{1}{d_{\min }}+\frac{2 \kappa^{+}}{\cos \phi^{+}}\right)$and $\mu_{0}^{-1}=1+2 d_{\min } \kappa^{-} \cos \phi^{+}$. In fact we will show that it holds when $\lambda(a)^{d_{\max }}<\mu(b)^{2 d_{\min }}$.

Hasselblatt and Schmeling in [HS] proposed the conjecture that equation 8.2 holds generically or under mild hypotheses, even for non-conformal diffeomorphisms and non-Lipschitz holonomies. They proved this conjecture for a class of Smale solonoids. If the conjecture is shown to be true, at least in the case of dynamical billiards, then we recover the equation 8.3 . If not, then the result still holds for 
the class of billiards in Sto1. We now calculate the constant $\alpha$ to get an estimate in terms of constants related to the billiard.

\section{Calculating the Hölder constant}

A combination of arguments from [Sto1, $\mathrm{H}$ ] and Section 11 can be used to calculate the Hölder constant $\alpha$ for the holonomies. The open billiard flow $S_{t}$ is an example of an Axiom A flow, with hyperbolic splitting into $T M=E^{s u} \oplus E^{s s} \oplus E^{S}$. These are the strong stable manifold, strong unstable manifold and the direction of the flow $S$ respectively. That is, for some $0<\eta<1$ we have $\left\|d S_{t}(u)\right\| \leq C \eta^{t}\|u\|$ for all $u \in E^{s}(t)$ and $t \geq 0$, and $\left\|d S_{t}(u)\right\| \leq C \eta^{-t}\|u\|$ for all $u \in E^{u}(t)$ and $t \leq 0$.

For each point $x$ there exist $\alpha_{x}<\beta_{x}<0<\alpha_{x}^{\prime}<\beta_{x}$ such that for $v \in E^{s s}(x)$, $u \in E^{s u}(x)$ and $t>0$ we have

$$
\begin{aligned}
& \frac{1}{C} e^{\alpha_{x} t}\|v\| \leq\left\|d S_{t}(x) \cdot u\right\| \leq C e^{\beta_{x} t}\|v\|, \text { and } \\
& \frac{1}{C} e^{-\alpha_{x}^{\prime} t}\|u\| \leq\left\|d S_{-t}(x) \cdot u\right\| \leq C e^{-\beta_{x}^{\prime} t}\|u\| .
\end{aligned}
$$

In the case of billiards, the reflection property $W_{\theta}^{(u)}=\operatorname{Refl} W^{(s)}(\operatorname{Refl}(x))$ implies that $\alpha_{x}=-\alpha_{x}^{\prime}$ and $\beta_{x}=-\beta_{x}^{\prime}$. The Hölder constant $\alpha$ is then given by the bunching constant $\alpha=B^{u}(S)=\inf _{x \in M_{0}} \frac{\beta_{x}-\beta_{x}^{\prime}}{\alpha_{x}}=\inf _{x \in M_{0}} \frac{2 \beta_{x}}{\alpha_{x}}[\mathrm{H}]$. The system is said to satisfy the pinching condition if there exist $0<\alpha_{0} \leq \beta_{0}$ such that $0 \leq \alpha_{0} \leq \alpha_{x}^{\prime} \leq \beta_{x}^{\prime} \leq \beta_{0}$ and $2 \alpha_{x}-\beta_{x} \geq \alpha_{0}$ for all $x \in M_{0}$.

Let $\hat{X}=S_{\tau}\left(W_{\theta}^{(u)}(x)\right)$ for some small $\tau$, let $t>d_{1}(x)+\ldots+d_{n}(x)$ and let $\delta_{j}(s)$ be defined as in section 4.3 . Then from [Sto1, there are constants $c_{1}, c_{2}$ such that

$$
\begin{aligned}
\frac{c_{1}}{c_{2}} \frac{\|u\|}{\delta_{1}(0) \delta_{2}(0) \ldots \delta_{n}(0)} & \leq\left\|d S_{t}(x) \cdot u\right\| \leq \frac{c_{2}}{c_{1}} \frac{\|u\|}{\delta_{1}(0) \delta_{2}(0) \ldots \delta_{n}(0)} \\
\frac{c_{1}}{c_{2}} \frac{\|u\|}{\mu_{0}^{n_{0}} \mu^{n-n_{0}}} & \leq\left\|d S_{t}(x) \cdot u\right\| \leq \frac{c_{2}}{c_{1}} \frac{\|u\|}{\lambda_{0}^{n_{0}} \lambda^{n-n_{0}}} . \\
\frac{c_{1}}{c_{2}}\left(\frac{\mu}{\mu_{0}}\right)^{n_{0}} \mu^{-t / d_{\max }}\|u\| & \leq\left\|d S_{t}(x) \cdot u\right\| \leq \frac{c_{2}}{c_{1}}\left(\frac{\lambda}{\lambda_{0}}\right)^{n_{0}} \lambda^{-t / d_{\min }}\|u\| \\
A e^{-t \ln \mu / d_{\max }}\|u\| & \leq\left\|d S_{t}(x) \cdot u\right\| \leq B e^{-t \ln \lambda / d_{\min }}\|u\|,
\end{aligned}
$$

where $\lambda=\lambda(b)=\frac{1}{1+d_{\max } b}, \mu=\mu(a)=\frac{1}{1+d_{\min } a}$, while $A=A(a, b)$ and $B=$ $B(a, b)$ are new global constants that exist for all $a<g_{\min }, b>g_{\max }$ (these are not necessarily bounded above). This inequality holds for all $t \geq t_{0}$ with $t_{0}$ sufficiently large that $m>n_{0}$, but there must be constants $A^{\prime}$ and $B^{\prime}$ such that the same inequality holds for all $0<t \leq t_{0}$. Taking $C$ large enough that $C>\max \left\{B, B^{\prime}\right\}$ and $\frac{1}{C}<\min \left\{A, A^{\prime}\right\}$, we now have $\alpha_{x}=-\ln \mu / d_{\max }$ and $\beta_{x}=-\ln \lambda / d_{\min }$ so the bunching constant is $B^{u}(S)=\frac{2 d_{\min } \ln \mu}{d_{\max } \ln \lambda}$. This argument improves Proposition 1.2 in [Sto1] by replacing $\left[\mu_{0}, \lambda_{0}\right]$ with the smaller interval $[\mu, \lambda]$ for any $a<g_{\min }, b>$ $g_{\text {max }}$.

Proposition 10.1. Let $a<g_{\min }, b>g_{\max }$. Assume that $\lambda(b)^{d_{\max }}<\mu(a)^{2 d_{\min }}$ and the boundary $\partial K$ is $C^{3}$. Then the open billiard flow in the exterior of $K$ satisfies the pinching condition on its non-wandering set $M_{0}$. For any $x \in M_{0}$ we can choose $\alpha_{x}=\alpha_{0}=\frac{\ln \mu(a)}{d_{\max }}$ and $\beta_{x}=\beta_{0}=\frac{\ln \lambda(b)}{d_{\min }}$. 
We cannot take the limit as $a \rightarrow g_{\min }, b \rightarrow g_{\max }$ for this proposition, since the constants $A$ and $B$ may not be bounded above. However when $\lambda^{d_{\max }}<\mu^{2 d_{\min }}$ we have $\alpha=1$ so equations 8.2 and 8.3 hold. Taking limits we can extend this to $\lambda_{1}^{d_{\max }}<\mu_{1}^{2 d_{\min }}$, which proves part 2 of the main theorem. If 8.2 does not hold then we have the following general estimate using (8.1):

$$
-\frac{4 d_{\min } \ln \mu_{1} \ln (u-1)}{d_{\max }\left(\ln \lambda_{1}\right)^{2}} \leq \operatorname{dim}_{H} M_{0} \leq-\frac{d_{\max } \ln \lambda_{1} \ln (u-1)}{d_{\min }\left(\ln \mu_{1}\right)^{2}}
$$

\section{ImPROVEMENT OF ESTIMATES}

11.1. Convex hull conjecture. We propose a conjecture that restricts the nonwandering set to a smaller area. This allows some relaxation of conditions.

Definition 11.1. For any $i \neq j$, let $\left(p_{i j}, p_{j i}\right) \in K_{i} \times K_{j}$ denote the minimum of $F: K_{i} \times K_{j} \rightarrow \mathbb{R},\left(q_{1}, q_{2}\right) \mapsto\left\|q_{1}-q_{2}\right\|$. Then each $p_{i j}$ is on the boundary $\partial K_{i}$ and the vector $p_{j i}-p_{i j}$ is normal to $\partial K_{i}$ at $p_{i j}$.

Conjecture 11.2. Denote the convex hull $\operatorname{Cvx}\left\{p_{i j}: 1 \leq i, j \leq n, i \neq j\right\}$ by $H$. Let $1 \leq \alpha_{1}, \ldots, \alpha_{n} \leq u(n \geq 3)$ be a finite sequence of indices and let $\left(q_{1}, \ldots, q_{n}\right)$ be a periodic billiard trajectory such that $q_{j} \in K_{\alpha_{k}}$ for each $j$. Then each $q_{j}$ is contained in $H$. Furthermore, the non-wandering set $M_{0}$ is contained in $H$.

We prove this conjecture for the case of an 3-dimensional billiard in which the obstacles are spheres. A very similar proof will work for all two-dimensional billiards, and higher dimensional billiards with hyperspherical obstacles. The general case in higher dimensions may be more difficult.

Proof of the conjecture for spherical obstacles. If the obstacles are spheres, then $H \cap Q$ is simply the convex hull of the centres of the spheres intersected with $Q$. Suppose that $\left(q_{1}, \ldots, q_{n}\right)$ is a periodic trajectory, but that at least one point is outside $H$. Without loss of generality we can number the points and obstacles such that $q_{1} \notin H$ and $\alpha_{1}=1$. $H$ is bounded by a number of planes, so $q_{1} \in K_{1}$ is on the outside (i.e. the side not containing $H$ ) of one such plane, say $\Pi=\Pi_{123}$, determined by the centres of obstacles $K_{1}, K_{2}, K_{3}$. Let $\nu$ be the outward normal vector of $\Pi$ and denote $v_{j}=\frac{q_{j+1}-q_{j}}{\left\|q_{j+1}-q_{j}\right\|}$, (with the convention that $q_{0}=q_{n}$ ). Without loss of generality, assume that $v_{0} \cdot \nu>0$.

For each $k \geq 1$ we have $q_{k+1}=q_{k}+d_{k} v_{k}$ and $v_{k}=v_{k-1}-2\left\langle v_{k-1}, n_{K}\left(q_{k}\right)\right\rangle n_{K}\left(q_{k}\right)$. We also have $\left\langle v_{k-1}, n_{K}\left(q_{k}\right)\right\rangle<0$. We show by induction that $q_{k} \cdot \nu>q_{1} \cdot \nu$ and $v_{k-1} \cdot \nu>v_{0} \cdot \nu$ for all $k>1$.

Suppose $q_{k} \in \partial K_{\alpha_{k}}$ is on the outside of $\Pi$ and $v_{k-1} \cdot \nu>v_{0} \cdot \nu$. The centre of $\partial K_{\alpha_{k}}$ is on the inside of $\Pi$, so the normal vector $n\left(q_{k}\right)$ must point away from $\Pi$, i.e. $n_{K}\left(q_{k}\right) \cdot \nu>0$. So $v_{k} \cdot \nu=v_{k-1} \cdot \nu-2\left\langle v_{k-1}, n_{K}\left(q_{k}\right)\right\rangle n_{K}\left(q_{k}\right) \cdot \nu>v_{k-1} \cdot \nu>v_{0} \cdot \nu$. Then $q_{k+1} \cdot \nu=q_{k} \cdot \nu+d_{k} v_{k} \cdot \nu>q_{k} \cdot \nu$. So $q_{k+1}$ is also on the outside.

For the orbit to be periodic we must have $q_{1}=q_{n+1}$ for some $n$. So by contradiction, all periodic points must be contained in $H$. Since $H$ is a closed set and the periodic points are dense in $M_{0}$, we have $M_{0} \subset H$.

Corollary 11.3 (Corollary 1). Given a billiard for which the above conjecture is true, the non-wandering set $M_{0}$ is entirely contained in $H$, which means any change to the billiard outside of $H$ will not have any effect on the non-wandering set, unless 
P. WRIGHT

it introduces a new periodic point. This means all results in this paper (and perhaps others) apply to billiards that are not smooth or convex, or that violate the no-eclipse condition $(\boldsymbol{H})$, provided that the intersection $K \cap H$ still satisfies these conditions.

Corollary 11.4 (Corollary 2). In cases where the conjecture is true, we can use the set $H$ to find better estimates for billiard constants. For example, we can estimate $d_{\max } \leq$ diam $H$. The minimum and maximum curvatures over $M_{0}$ can be estimated by $\kappa^{-} \leq \min _{q \in \partial K \cap H} \kappa_{\min }(q)$ and $\kappa^{+} \leq \min _{q \in \partial K \cap H} \kappa_{\max }(q)$.

11.2. Adjusted domain of $g$. Recall that the natural domain for the function $g$ is $\left[\kappa^{-} \cos \phi^{+}, \frac{\kappa^{+}}{\cos \phi^{+}}\right] \times\left[d_{\min }, d_{\max }\right]$. This applies in billiards where the dimension $D>2$; when $D=2$ the natural domain is $\left[\kappa^{-}, \frac{\kappa^{+}}{\cos \phi^{+}}\right] \times\left[d_{\min }, d_{\max }\right]$ (see the end of section 4.2. To cover both cases at once, we let $\iota=0$ if $D=2$ and $\iota=1$ if $D>2$, so that $\cos ^{\iota} \phi$ is 1 if $D=2$ and $\cos \phi$ otherwise. Define the adjusted domain by

$$
\mathbb{D}=\bigcup_{i, j}\left[\kappa_{i}^{-} \cos ^{\iota} \phi_{i j}^{+}, \frac{\kappa_{i}^{+}}{\cos \phi_{i j}^{+}}\right] \times\left[d_{i j}^{-}, d_{i j}^{+}\right]
$$

where $\kappa_{i}^{-}, \kappa_{i}^{+}$are the minimum and maximum curvatures on $\partial K_{i} \cap H, d_{i j}^{-} \geq$ $\left|p_{i j}-p_{j i}\right|, d_{i j}^{+} \leq \max _{k, l}\left|p_{i k}-p_{j l}\right|$ are the minimum and maximum distances between $K_{i} \cap H$ and $K_{j} \cap H$, and $\phi_{i j}=\max \left\{\phi(x): x \in K_{i} \cap H, B x \in K_{j} \cap H\right\}$ is the maximum collision angle over trajectories from $K_{i}$ to $K_{j}$. These $\phi_{i j}$ can be estimated by $\cos \phi_{i j} \geq \frac{b_{i j}^{-}}{d_{\max }}$ where $b_{i j}^{-}=\min _{k} d\left(K_{i}, C v x\left(K_{j}, K_{k}\right)\right)$.

The minimum and maximum values of $g$ over the natural domain may be outside of the adjusted domain. The minimum and maximum values in the adjusted domain are given by

$$
\begin{aligned}
& g_{\min }=\min \{g(\gamma, \theta):(\gamma, \theta) \in \mathbb{D}\}=\min \left\{g\left(\kappa_{i}^{-} \cos ^{\iota} \phi_{i j}, d_{i j}^{+}\right), 1 \leq i, j, \leq u\right\} \\
& g_{\max }=\max \{g(\gamma, \theta):(\gamma, \theta) \in \mathbb{D}\}=\max \left\{g\left(\frac{\kappa_{j}^{+}}{\cos \phi_{i j}}, d_{i j}^{-}\right), 1 \leq i, j, \leq u\right\}
\end{aligned}
$$

Lemma 11.5. For any $x=(q, v) \in M_{0}$, we have $\left(\frac{\kappa\left(q_{j}\right)}{\cos \phi_{j}(x)}, d_{j}(x)\right) \in \mathbb{D}$ and $\left.\left(\kappa\left(q_{j}\right) \cos ^{\iota} \phi_{j}(x)\right), d_{j}(x)\right) \in \mathbb{D}$ for all $j \in \mathbb{Z}$.

Proof. Assume $D>2$. Since $q_{j}=\pi B^{j} x \in M_{0}$ for all $j \in \mathbb{Z}$, we have $\kappa\left(q_{j}\right) \in$ $\left[\kappa_{i_{j}}^{-}, \kappa_{i_{j}}^{+}\right], \phi\left(B^{j} x\right) \in\left[0, \phi_{i_{j} i_{j+1}}^{+}\right]$, and $d\left(q_{j}, q_{j+1}\right) \in\left[d_{i_{j} i_{j+1}}^{-}, d_{i_{j} i_{j+1}}^{+}\right]$. Hence there exist some integers $1 \leq a, b, \leq u$ such that $\kappa\left(q_{j}\right) \cos \phi\left(B^{j} x\right) \geq \kappa_{a}^{-} \cos \phi_{a b}^{+}$and $\frac{\kappa\left(q_{j}\right)}{\cos \phi\left(B^{j} x\right)} \leq$ $\frac{\kappa_{a}^{+}}{\cos \phi_{a b}^{+}}$. For the same $a, b$ we have $d\left(q_{j}\right) \in\left[d_{a b}^{-}, d_{a b}^{+}\right]$. The proof for $D=2$ is analogous.

Example 11.6. Consider the billiard displayed in Figure 1 consisting of three disks arranged in an isoceles triangle of height 10 and base length 8 . The disks $K_{1}, K_{2}, K_{3}$ have radii 1,2 and 3 respectively. The solid lines give the distances $d_{i j}^{-}$ and the dashed lines give the distances $d_{i j}^{+}$. Figure 2 displays the adjusted domain over the natural domain, with contour lines of the function $g(\gamma, \theta)$. The following calculations were obtained using the programs Geogebra and Mathematica. 
Using the adjusted domain rather than the natural domain means that the interval $\left[g_{\min }, g_{\max }\right]$ is reduced from $[0.760,7.34]$ to $[0.762,3.41]$. Using the natural domain we have the estimate

$$
0.326 \leq \operatorname{dim}_{H} M_{0} \leq 1.167,
$$

but with the adjusted domain we get

$$
0.396 \leq \operatorname{dim}_{H} M_{0} \leq 1.165 .
$$

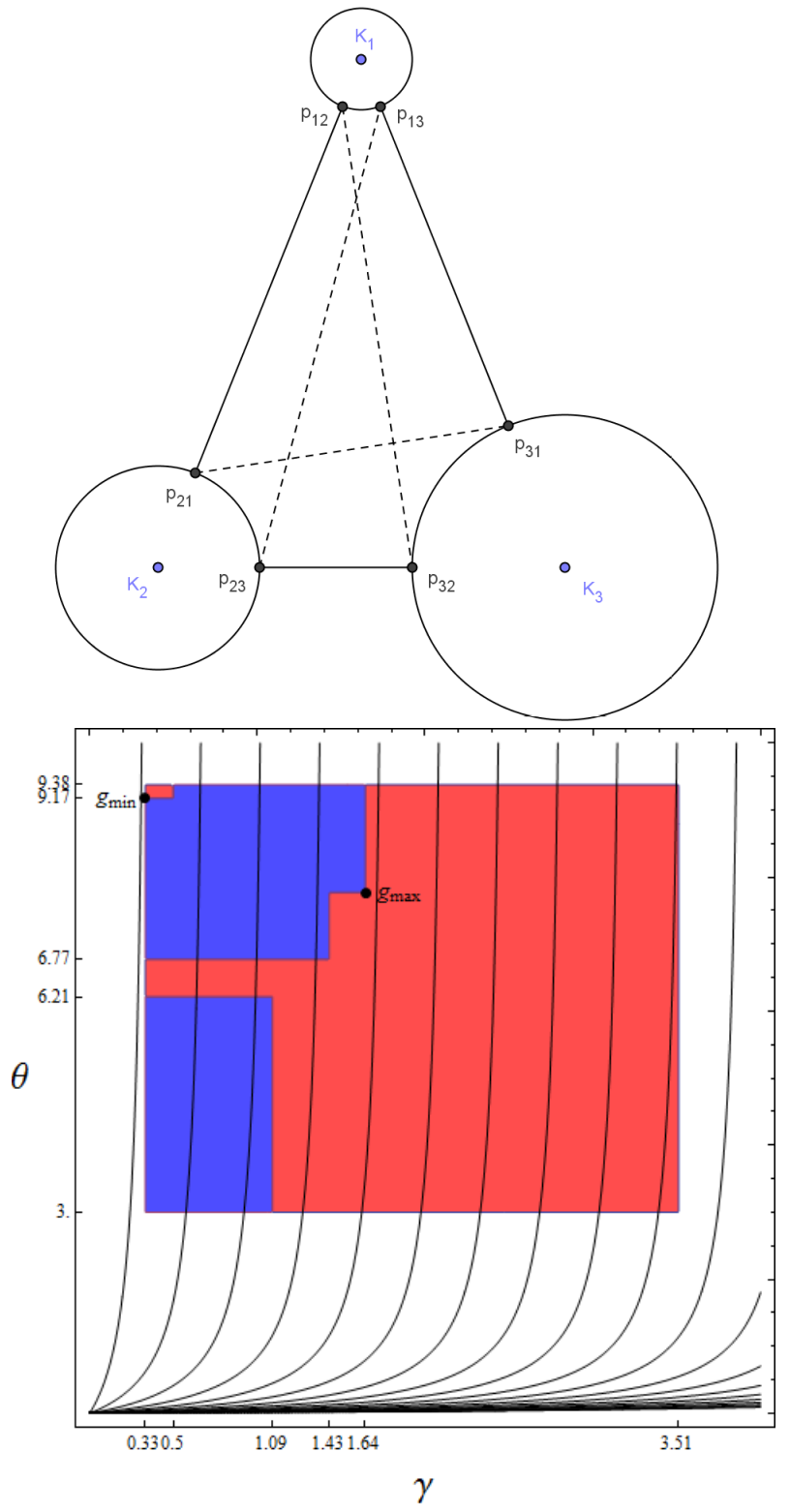

\section{REFERENCES}

[B] L. Barreira, A non-additive thermodynamic formalism and applications to dimension theory of hyperbolic dynamical sytems. Ergod. Th. \& Dynam. Sys. 16(1996), 871-927. 
[BCST] P. Bàlint, N. Chernov, D. Szász and I. P. Tóth. Geometry of multi-dimensional dispersing billiards. Astérisque 286(2003), 119-150.

[BPS] L. Barreira, Y. Pesin, J. Schmeling, Dimension and Product Structure of Hyperbolic Measures, Ann. of Math. 149(1999) no. 3, 755-783.

[Ch] N. Chernov, R. Markarian, Chaotic Billiards, American Mathematical Society, Mathematical Surveys and Monographs, 127(2006).

[Ed] G. Edgar, Measure, topology and fractal geometry. Springer-Verlag, New York, 1990.

[Fa] K. Falconer, Fractal Geometry, Wiley (2003).

[H] B. Hassleblatt, Regularity of the Anosov splitting II. Ergod. Th. \& Dynam. Sys. 17(1997), 169-172.

[HS] B. Hasselblatt, J. Schmeling, Dimension product structure of hyperbolic sets, Electon. Res. Announc. Amer. Math. Soc. 10(2004), 88-96.

[Ke] R. Kenny, Estimates of Hausdorff Dimension for the Non-Wandering Set of an Open Planar Billiard, Canad. J. Math. 120(2004), 115-133.

[M] T. Morita, The symbolic representation of billiards without boundary condition. Trans. Amer. Math. Soc. 325(1983), 819-828.

[P] Y. Pesin, Dimension theory in Dynamical Systems, Contemporary Views and Applications, Chic. Lect. in Math. (1997).

[S] Ya. G. Sinai, "Dynamical Systems with Elastic Reflections", Russian Math. Surveys, 25(1970), 137-191.

[Sjö] Johannes Sjöstrand, Geometric bounds on the density of resonances for semiclassical problems. Duke Math. J. 60(1990), 1-57.

[Sto1] L. Stoyanov, Non-integrability of Open Billiard Flows and Dolgopyat Type Estimates, Ergod. Th. and Dynam. Sys. doi:10.1017/S0143385710000933

[Sto2] L. Stoyanov, An estimate from above of the number of periodic orbits for semi-dispersed billiards, Commun. Math. Phys. 124(1989), 217-227

[Sto3] L. Stoyanov, Spectrum of the Ruelle operator and exponential decay of correlations for open billiard flows, Amer. J. Math. 123(2001), 715-759

Mathematics Department, University of Western Australia, Western Australia

E-mail address: paul.e.wright@uwa.edu.au 